\title{
El cuerpo como texto del dolor: cinco relatos de mujeres que han vivido violencia sexual en la ciudad de Medellín ${ }^{*}$
}

\author{
Natalia Mejía Acevedo** \\ Viviana Maritza Vargas Restrepo ${ }^{* * *}$
}

Recibido: 27 de enero de 2017

Enviado a pares evaluadores: 1 de febrero de 2017

Aprobado por pares evaluadores: 10 de marzo de 2017

Aprobado por comité editorial: 20 de abril de 2017

DOl: 10.22395/csye.v6n11a2

\section{RESUMEN}

Este artículo integra el resultado de una investigación que tuvo como propósito indagar por las percepciones que tienen de su cuerpo cinco mujeres de la ciudad de Medellín que han vivido violencia sexual y han sido atendidas por el Centro de Recursos Integrales para la Familia -CERFAMI- en el período comprendido entre 2010-2016, explorando los cambios que se evidencian en la relación con el propio cuerpo antes y después del hecho violento. En el estudio se realizó un proceso dialógico desde la pers- pectiva feminista, donde se tuvo acceso a un conjunto de experiencias del dolor y del abuso sobre la corporalidad femenina que permitieron reflexionar sobre la condición encarnada de subjetividades violentadas. Por lo tanto, cada uno de los testimonios de las mujeres que participaron en esta investigación integra el tejido sensible y el compromiso político y ético de este artículo.

Palabras clave: violencia sexual (VS), cuerpo, identidad de género, experiencias encarnadas del dolor, subjetividad.

Este artículo proviene de un proyecto de investigación, el cual lleva por título: Proceso de resignificación del cuerpo en mujeres abusadas sexualmente que han sido atendidas por el Centro de Recursos Integrales para la Familia -CERFAMI-. Los nombres que se utilizaron en el artículo para cada una de las cinco mujeres fueron seudónimos, que relacionamos con personajes de la literatura latinoamericana y colombiana, la mitología y la religión; cada uno de estos seudónimos tiene un significado que lo relacionamos con la personalidad de cada mujer. Esta investigación fue asesorada por el docente de la Universidad de Medellín, Hilderman Cardona-Rodas.

** Antropóloga de la Universidad de Antioquia y Especialización en Estudios de Género de la Universidad de Medellín. Correo electrónico: natalia.mejia3421@gmail.com

*** Licenciada en Educación Básica con énfasis en Tecnología e Informática de la Universidad Católica Luis Amigó, Especialista en Intervenciones Psicosociales de la Universidad Católica Luis Amigó y Especialización en Estudios de Género de la Universidad de Medellín. Correo electrónico: vivitos91@gmail.com 


\section{The body as a text of pain: five stories of women who have experienced sexual violence in the city of Medellín}

\section{ABSTRACT}

This article integrates the result of an

to have access to a series of experiences of investigation that had as a purpose search the perceptions about the body, of five Medellin city women that had experienced sexual violence and were attended by the Center of Integral Resources for the Family CERFAMI in the Period between 2010-2016, exploring the changes that are evidenced in the relationship with the body itself before and after the violent event. In the study, a dialogical process was carried out pain and abuse on the feminine corporality that allowed to reflect on the incarnated condition of violated subjectivities. Therefore, each of the testimonies of women who participated in this research integrate the sensitive background and the political and ethical commitment of this article.

Key words: sexual violence (VS), body, gender identity, incarnated experiences of pain, subjectivity. 


\section{Introducción}

Resignificar el cuerpo, hacerlo parte de un nuevo registro vital para comprender otras dimensiones del espacio sensible que somos en lo humano, constituye una experiencia de reinvención en la cual se trascienden los dolores y las huellas que impregnan de terror la relación con la existencia. La humillación de un cuerpo abusado, y más aún, de un cuerpo ultrajado sexualmente hace sentir la vida como un tormento en los recuerdos y marcas; un suplicio que, para el caso de las mujeres, implica una fuerza de voluntad para recrear su propio cuerpo.

Desde este preámbulo, la investigación indagó por las percepciones que tienen de su propio cuerpo las mujeres que han vivido VS y que han sido atendidas en el Centro de Recursos Integrales para la Familia -CERFAMI-, entidad fundada en el año 1989, la cual tiene como función promover la salud sexual y reproductiva, en particular con aquellas personas que, en razón de su sexo, edad, posición o condición social, han sido víctimas de exclusión, violencia o discriminación.

Desde los años sesenta del siglo XX, una pregunta por el cuerpo emerge desde el problema de la autonomía, la salud, la política y la justicia social. En este contexto las mujeres colombianas ya tenían acceso a la Educación Superior, momento en el cual un grupo de mujeres antioqueñas, de diferentes profesiones y oficios, comenzaron a cuestionar la condición y la posición de las mujeres en la sociedad colombiana, consolidando con ello un movimiento social de mujeres que bebió del feminismo, de los derechos humanos y de la creciente preocupación por los derechos sexuales y reproductivos y de salud sexual imperantes en la época, en tanto consignas políticas de lucha. Luego del Primer Encuentro Feministas Latinoamericano, realizado en Bogotá en 1981, varios colectivos de mujeres feministas se fortalecieron en el país, agrupadas con diferentes nombres y teniendo como característica principal la diversidad de posturas, condiciones y posiciones de las participantes. En Medellín, a principios de la década del setenta, surgió el primer grupo de mujeres dedicado a estudiar la condición de la mujer en el país, formando el primer centro de documentación sobre estudios feministas y realizando tertulias sobre estos temas. Este grupo, conformado por cinco mujeres de diferentes profesiones, entre ellas, Dilia Rodríguez, cofundadora y actual presidenta de la junta de CERFAMI, se denominó Centro de Estudios sobre la mujer (CEM), que jugó un papel relevante en las actividades de celebración del Año Internacional de la Mujer, iniciativa de las Naciones Unidas en el año 1975, así como en la difusión del Primer Plan de Acción Mundial acordado en la Primera Conferencia Internacional de la Mujer, celebrada en la ciudad de México ese mismo año. El grupo CEM continuó trabajando varios años y se sumó a las acciones en torno al Decenio de las Naciones Unidas para la Mujer (1975-1985). 
En esta trama sociopolítica, algunas mujeres de Medellín, especialmente médicas, abogadas, enfermeras y psicólogas, enfatizaron en los temas referidos a la salud sexual y reproductiva de las mujeres y en los derechos que los sustentan. Es así como se nutre una de las primeras organizaciones mixtas feministas que llevó el nombre Corporación Salud Mujer que nació en 1986. Carmen Posada y María Cecilia Alzate lideraron, para ese entonces, la fundación de la Corporación Servicios Alternativos para la Mujer, acompañadas de un grupo de profesionales de las áreas sociales y de la salud, interesado en hacer realidad los derechos sexuales y reproductivos de las mujeres. Corporación Salud Mujer realizó un trabajo valioso por la promoción de los derechos humanos y los derechos sexuales y reproductivos de las mujeres, así como en la atención en salud, trabajo que fue reconocido en al ámbito nacional e internacional. En el año 1988, con motivo del Día Internacional de la Mujer, el Concejo de Medellín hizo a Corporación Salud Mujer un reconocimiento por su compromiso con las mujeres de la cuidad. Infortunadamente, a finales de 1988, cuando fuerzas oscuras opuestas a la vigencia de los derechos humanos asolaron la cuidad y el país con amenazas y asesinatos, Salud Mujer se vio obligada a cerrar sus puertas para proteger la vida y la integridad de su directora y de sus integrantes, ante las graves amenazas. Muchas de aquellas mujeres que fundaron esa corporación siguieron liderando procesos de reivindicación política y social de las mujeres que derivaron en el nacimiento en 1989 de Centro de Recursos Integrales para la Familia (CERFAMI) ${ }^{1}$.

En memoria de tan significativa labor, signada por la voluntad, el esfuerzo y la convicción de no silenciar el abuso en ninguna de sus manifestaciones y consecuencias, se hace necesario retornar siempre a la palabra como una fuente estética para resignificar y reinventar las historias de vida que han tenido el cuerpo como relato de dolor, derivado de acontecimientos de violencia sexual.

Desde esta postura, para esta investigación se realizaron entrevistas semiestructuradas a cinco mujeres de la ciudad de Medellín, entre los 23 y 52 años y asistentes al programa de hogares de acogida o asesoría terapéutica de CERFAMI que han sufrido VS, quienes voluntariamente aceptaron ser entrevistadas para que fueran analizadas sus vivencias y cómo se han reflejado en el ámbito corporal. La presente es una investigación que aporta a la definición de una

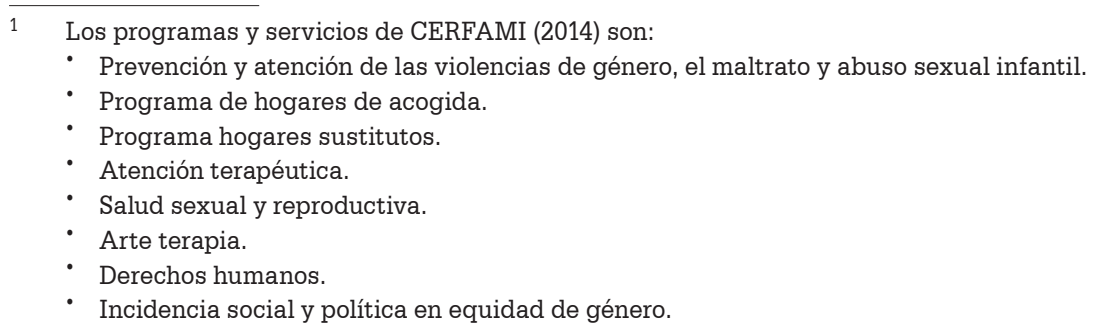


propuesta de intervención para mujeres que han sido abusadas sexualmente, la cual incorpora la rehabilitación del cuerpo abusado, componente que no ha sido tenido en cuenta en las rutas de atención. El artículo aborda los cambios que tuvieron lugar en la percepción del cuerpo de mujeres agredidas sexualmente y da cuenta de los efectos emocionales y corporales que el evento traumático ha tenido en su vida.

Esta investigación tiene una perspectiva feminista, ya que, como afirman Deysy Zuluaga y Alfonso Insuasty (2011), es un estudio de las mujeres, con una mirada crítica que conduce a un mecanismo de liberarlas de una opresión patriarcal inmersa en sus cuerpos; además, porque se aborda a las mujeres en una doble dimensión, por una parte, como sujeto de investigación y, por otra parte, como objeto de estudio; incorpora una de las tres tendencias teóricas de los Estudios de las Mujeres citado por M. José Guerra (1999), a saber, re-acción y / o re-visión, cuyo objetivo sería: "El estudio de la opresión de las mujeres -roles genéricos-, desigualdades, discriminación, exclusión; sus causas, naturaleza y efectos; la percepción de las mujeres de la injusticia y su respuesta a ella" (p. 47).

En este artículo se tratan de visibilizar las perspectivas que tienen estas cinco mujeres de sus cuerpos después de sufrir VS, además de analizar cómo influye este suceso en sus vidas cotidianas y la importancia que debe tener este aspecto en una ruta de atención a mujeres que han sufrido VS. Para la recolección de información utilizamos la metodología feminista que, como lo señala Sandra Harding (1998), las investigadoras feministas:

- Escuchan muy atentamente lo que las mujeres informantes piensan acerca de sus propias vidas y de las de los hombres.

- Mantienen posiciones críticas frente a las concepciones de los científicos sociales tradicionales sobre las vidas de hombres y mujeres.

- Observan también algunos comportamientos de mujeres y hombres que, desde la perspectiva de los científicos sociales tradicionales, no son relevantes.

- En el caso de la historia, buscan patrones de organización de los datos históricos no reconocidos con anterioridad (p. 47).

Este artículo está dividido en tres capítulos: en el primer apartado el análisis se orienta hacia la condición corporal de la experiencia humana y su relación con la construcción de la identidad de género y la violencia contra las mujeres; posteriormente se examinan las subjetividades encarnadas de la violencia y, por último, se presenta una reivindicación del coro de voces que conformaron esta investigación. 


\section{Testimonios corporales de la violencia}

El cuerpo es el protagonista de la experiencia dolorosa que comparten las mujeres que han vivido VS; en él se manifiestan las heridas, sufrimientos, percepciones, emociones y sentimientos que derivan y conforman un testimonio de la misma. Las mujeres que han experimentado estos acontecimientos violentos reconocen una afectación en el cuerpo desde diferentes dimensiones: la imagen, el significado y el uso.

La imagen emerge a partir de la concepción de construcciones compartidas de género, convocando formas de auto-concepción diferentes, por lo que se inicia una observación negativa de sí misma. El significado reconoce la destrucción de concepciones que hasta entonces había adquirido, filtrándose sentidos negativos que se reflejan en críticas y culposas ideas. El uso de su cuerpo como entidad estética y disfrute propio de la sexualidad empieza a generar modificaciones que esbozan la aparición de restricciones y aislamiento. El rol empieza a cambiar y poco a poco, cada palabra, cada forma de auto-observación y usos se va dejando a la construcción individual de cada mujer.

Cuando el cuerpo es fuente de experiencias dolorosas, se habla de lo reprimido. ¿Cómo el cuerpo reacciona frente a lo reprimido?, ¿cómo este eje de sexualidad y lenguaje reacciona frente a un abuso sexual?, ¿qué sentido tiene el cuerpo para una mujer abusada sexualmente? Estos son algunos interrogantes que se quieren exponer en esta investigación, y para esto es necesario abordar el significado de cuerpo en las mujeres, ya que estas son quienes cargan con una serie de concepciones inmersas en normas, valores, juicios morales, culpas, miedos, prejuicios y estereotipos por su condición de género, entre otros.

Para explicar el concepto de cuerpo, es preciso remitirse al habitus que Pierre Bourdieu (2007) [1980] presenta como historia hecha cuerpo, es decir, las estructuras sociales que el ser humano encarna a lo largo de su vida

Los condicionamientos asociados a una clase particular de condiciones de existencia producen habitus, sistemas de disposiciones duraderas y transferibles, estructuras estructuradas predispuestas para funcionar como estructuras estructurantes, es decir, como principios generadores y organizadores de prácticas y representaciones que pueden estar objetivamente adaptadas a su fin sin suponer la búsqueda consciente de fines y el dominio expreso de las operaciones necesarias para alcanzarlos, objetivamente "reguladas" y "regulares" sin ser el producto de la obediencia a reglas, y, a la vez que todo esto, colectivamente orquestadas sin ser producto de la acción organizadora de un director de orquesta (p. 92).

El habitus remite a la manera en que las personas inscriben en su cuerpo lo social y a un principio que sustenta las prácticas culturales de un contexto específico. Sobre este concepto, Galak (2010) indica que la subjetividad, lo 
individual, etc., son construcciones sociales y no un producto de la naturaleza, "sino que, al igual que el cuerpo, son construcciones simbólicas. De allí que se entienda al habitus como estructuras estructuradas predispuestas a funcionar como estructuras estructurantes y no como estructuras estructuradas y estructurantes" (p. 29), lo cual afirma que obedecen a su potencial transformador y a la potencia limitada del cuerpo, sobre el cual recaen las limitaciones que le impone el espacio social en razón de su biología, pero, a su vez, es posibilidad por ser productor de significados sociales.

Galak (2010) explica que, según Bourdieu, el habitus se manifiesta de forma práctica en la hexis corporal, término con el que hace alusión a la expresión de los agentes, la cual conlleva significados y valores, se moldea a partir de la educación recibida desde la infancia y conforma una serie de hábitos que generan en el individuo una memoria corporal que se expresa en el modo de caminar, comer, gesticular, etc.

Bourdieu (2000) [1998] introduce el concepto de dominación masculina para explicar el orden sociocultural que favorece las relaciones desiguales entre hombres y mujeres, así:

El trabajo de construcción simbólico no se reduce a una operación estrictamente performativa de motivación que orienta y estructura las representaciones, comenzando por las representaciones del cuerpo (lo que no es poca cosa); se completa y se realiza en una transformación profunda y duradera de los cuerpos (y de los cerebros), o sea, en y a través de un trabajo de construcción práctico que impone una definición diferenciada de los usos legítimos del cuerpo, sexuales sobre todo, que tiende a excluir del universo de lo sensible y de lo factible todo lo que marca la pertenencia al otro sexo -y en particular todas las virtualidades biológicamente inscritas en el "perverso polimorfo" que es, de creer a Freud, cualquier niño-, para producir ese artefacto social llamado un hombre viril o una mujer femenina. El nomos arbitrario que instituye las dos clases en la objetividad solo reviste la apariencia de una ley natural (se habla corrientemente de sexualidad o, incluso en la actualidad, de matrimonio "contra natura") al término de una somatización de las relaciones sociales de dominación. Solo a cambio y al término de un formidable trabajo colectivo de socialización difusa y continua las identidades distintivas que instituye el arbitrario cultural se encarnan en unos hábitos claramente diferentes de acuerdo con el principio de división dominante y capaces de percibir el mundo de acuerdo con ese principio (pp. 37-38).

Estos esquemas se perpetúan mediante la violencia simbólica sobre los cuerpos, y su fundamento radica en lo biológico, es decir, en la división masculino / femenino, la cual es considerada como algo objetivo y natural, lo que conlleva la justificación de dichas diferencias sociales y a la consecuente dominación masculina. Existe entonces un poder simbólico en los agentes sociales que ejercen la violencia sobre otros con su complicidad, ya que esta es aceptada y se fundamenta en el desconocimiento de su modo de operar; por lo tanto, el 
dominado observa la relación de dominación desde las mismas categorías del dominador.

El habitus es la historia hecha cuerpo. Al respecto, Galak (2010) indica que para Bourdieu, la historia es naturalizada, incuestionable e ignorada como tal debido al trabajo de instituciones como la familia, la iglesia, el Estado y la escuela, lo que las convierte en fuerzas que conducen al olvido de la misma; por esto, la arbitrariedad cultural deriva en algo natural y deja en evidencia los mecanismos mediante los cuales se ejerce la dominación de los hombres sobre las mujeres.

Igualmente es necesario examinar los aportes de Elisa Seoane (2015) a partir de su lectura de Bourdieu, entre los que se destaca su concepto de habitus de género primario, el cual emplea para describir la acción pedagógica de la familia y la escuela al incorporar en las personas desde su infancia un modo de actuar y de percibir el mundo de una determinada manera mediante refuerzos positivos y negativos, mientras que el repertorio de género constituye la serie de comportamientos y formas de conducirse en la vida que se enseña a niños y niñas durante su socialización; por lo tanto, en los primeros fomenta la independencia, la agresividad y el alejamiento de las conductas que denotan afecto; en cambio, sobre las segundas, se impulsa la dependencia, la expresión de sus sentimientos y la atención de las necesidades de los demás por encima de las suyas.

Para Seoane (2015), el habitus de género es decisivo en la conformación de la identidad de género porque "permite comprender cómo se construye el género, al ser definido como esquemas incorporados que dan estructura a las conductas, actitudes y formas de sensibilidad que hacen que una persona se sienta y perciba como masculina o femenina" (pp. 110-111). Al respecto, la autora llama la atención sobre la imagen fuertemente estereotipada de la feminidad, la cual ha hecho de las mujeres un "... testigo hostil del ser corporal, de ahí que llegue a observarse con un cuerpo inferiorizado, pues la imagen corporal que se presenta en los medios sugiere una belleza femenina perfecta y, por ello, inalcanzable para la mayoría de las mujeres" (p. 112).

Según Galak (2010), el concepto de cuerpo alienado, de Pierre Bourdieu, remite a la incomodidad que experimenta una persona con su propio cuerpo debido a que no corresponde con lo que socialmente se espera de él; por lo tanto, el malestar, la vergüenza y la timidez se incrementan conforme más se aleje este del ideal colectivo; además, debe señalarse que el cuerpo socialmente exigido varía según el sexo y el contexto cultural.

Las huellas de la VS sobre las mujeres que participaron en esta investigación configuran cambios corporales que riñen con las concepciones que 
poseen desde su niñez sobre la manera en que deben presentarse en sociedad, el arreglo personal, el peso, la postura y la manera de interactuar con otras personas. Estos factores que según el contexto cultural se determinan con precisión para hombres y mujeres, de acuerdo con los roles asignados desde el nacimiento y los imaginarios de belleza imperantes, cambian después del hecho violento, generándose valoraciones diferentes de sí mismas y de su lugar en la sociedad.

El concepto de hexis corporal femenina que ofrece Seoane (2015) es definido como el lenguaje del cuerpo, el cual incluye los movimientos, las expresiones y los gestos de una persona; sin embargo, para las mujeres lleva implícita una ambigüedad porque su vivencia oscila entre el puedo y no puedo, ya que forman parte del mundo pero su cuerpo no se apropia ni se conduce por el mismo con la firmeza y seguridad con que lo hacen los hombres. Según la autora, la hexis corporal femenina conlleva altas dosis de pasividad, lo que constituye un importante rasgo del erotismo sexual, lo cual tiene un papel relevante en la VS que se ha ejercido contra las mujeres.

Cuando el cuerpo de las mujeres es violentado sexualmente, el lenguaje corporal se modifica puesto que se convierte en un testimonio del dolor que se ha apropiado de él, como lo señala Hildeman Cardona Rodas (2017):

Así, los actos violentos dejan huella sobre el cuerpo, en la relación entre persona, cuerpo y sociedad, en rituales encarnados en el ser-en-el-mundo, ya que la realidad social se halla impresa en el cuerpo. La experiencia corporal de la violencia se proyecta como lenguaje en los signos visibles del cuerpo violentado, asesinado, destrozado o humillado dejando tras de sí temores, represiones, miedos y exclusiones que se encarnan en los testimonios visuales del cuerpo despojo que ha sido deshumanizado y animalizado (pp. 106-107).

Por lo tanto, puede decirse que la corporalidad de las mujeres violentadas sexualmente fue desprovista de su subjetividad al haber sido esta reducida a simple objeto, donde el uso social de su cuerpo, en este orden social donde la dominación masculina es naturalizada, fue determinado por el agresor como un lugar para su satisfacción sexual, dejando como resultado las huellas del dolor.

Para Alicia Puleo (2015), una cosa y un ser humano se diferencian en que la primera es en sí, es decir, se caracteriza por ser algo definido, mientras que el segundo, se va construyendo a sí mismo a lo largo de su existencia, lo que le da entonces el carácter de ser para sí. La VS manifiesta una relación entre una cosa y una persona; de hecho, la autora expresa que las mujeres se convirtieron en objetos al imponerles roles (que se ejecutan siempre en función de otros) que les impedían decidir con libertad el rumbo de sus vidas. 
En su análisis sobre el cuerpo y la violencia, Puleo (2015) sostiene que la tendencia de unificar deseo sexual con dominación y destrucción como característica del poder masculino ha hecho que tanto la violencia como la crueldad, en vez de ser vistas como una desviación o un crimen, sean caracterizadas como algo natural y propio del lugar que se ocupe en la jerarquía social; sostiene entonces que dicha concepción surge de la construcción de la virilidad, lo cual se hace a partir de la negación de lo emocional y lo débil. " $\mathrm{El}$ 'duro' es un resultado de técnicas de género específicas que proceden a extirpar características previamente definidas como propias del sexo femenino. La construcción del héroe es una peligrosa empresa que no siempre resulta exitosa y puede fácilmente producir villanos" (Puleo, 2015, pp. 127-128).

La persona transformada en simple objeto durante la agresión constituye una trayectoria individual desechada y borrada; todo aquello que siente, piensa y desea el sujeto asociado a esa corporalidad específica es anulado. Para Lluís Duch y Joan-Carles Mèlich (2005),

La corporeidad no es nada más que un "espacio de vida móvil", en y sobre el cual se concreta, se salva o se pierde, se "fisionomiza" la limitada cantidad de espacio y de tiempo del que dispone cada individuo humano... Debería evitarse que se considerara al cuerpo humano como un simple "objeto" con la disponibilidad y la capacidad de manipulación que son propias de los meros objetos (p. 236).

Según estos autores, la diferencia entre cuerpo y corporeidad radica en que al hablar del cuerpo, se está nombrando simplemente un objeto, lo cual no es posible debido a su condición humana; en cambio, la corporeidad refiere a "alguien que posee conciencia de su propia "vivacidad", de su presencia aquí y ahora, de su procedencia del pasado y de su orientación hacia el futuro, de sus anhelos de infinito a pesar de su congénita finitud" (Duch, Mèlich, 2005, p. 240).

Respecto a lo anterior, David Le Breton (1992) afirma que el cuerpo es moldeado por el contexto cultural que habitan las personas; a través de este es posible relacionarse con el mundo, transformar el entorno, expresar los sentimientos, percibir los sucesos que acontecen y expresar símbolos de identidad. El aprendizaje de las modalidades corporales resultantes de la interacción de los seres humanos con su entorno conlleva la integración de los roles establecidos en el orden social.

La violencia sexual contra la mujer como asalto al cuerpo, a la identidad y a la dignidad, pertenece a un tipo de violencia de género, que evidencia las relaciones de dominación y subordinación aún presentes en nuestras sociedades, es uno más de los referentes para visibilizar la situación diferencial en la que se encuentran hombres y mujeres, además, de reflejar discriminación y constante violación de los derechos humanos (Pérez, 2010, p.13). 
Los cuerpos de las mujeres al estar situados en el tiempo y en el espacio, incorporan el discurso legitimado en la cultura donde se desarrollan sus vivencias; por lo tanto, debe resaltarse que,

Colombia ha sido un país de cultura patriarcal por tradición, el dominio del poder, los gobiernos, la economía y todas las demás relaciones sociales han estado en cabeza de los hombres, han sido ellos quienes tomen las decisiones que han de regir la vida en comunidad y es por esta razón que la historia los ha privilegiado al brindarles la potestad para interceder de manera directa en todas las cosas y escenarios que afecten a esa sociedad. Esta situación, en suma con los binarismos y divisiones de género según los criterios biológicos y psiquiátricos, deja como consecuencia que la masculinidad sea concebida como una sola, donde no caben otro tipo de manifestaciones y donde por supuesto se da una hegemonía heterosexual (Cardona, Sánchez, 2012, p. 47).

Los cuerpos de varones y hembras inscritos en el binario hombre-masculino y mujer-femenina hacen referencia a las maneras de comportarse reguladas socialmente, dado que la masculinidad y la virilidad configuran el modelo hegemónico; cuerpos como los de las mujeres y las personas cuyas performatividades de género son consideradas ilegítimas adquieren la condición de débil, pasivo, prohibido, anormal y se tornan susceptibles de sufrir violencia por su condición de diferente / dominado.

\section{Los mensajes del dolor}

Esta investigación tuvo como objetivo principal conocer las percepciones que tienen sobre sus cuerpos cinco mujeres habitantes de la ciudad de Medellín que han sido atendidas por CERFAMI; por lo tanto, se abordará el concepto de violencia sexual como

[...] el sexo bajo coacción de cualquier tipo incluyendo el uso de fuerza física, las tentativas de obtener sexo bajo coacción, la agresión mediante órganos sexuales, el acoso sexual incluyendo la humillación sexual, el matrimonio o cohabitación forzados incluyendo el matrimonio de menores, la prostitución forzada y comercialización de mujeres, el aborto forzado, la denegación del derecho a hacer uso de la anticoncepción o a adoptar medidas de protección contra enfermedades, y los actos de violencia que afecten a la integridad sexual de las mujeres tales como la mutilación genital femenina y las inspecciones para comprobar la virginidad (Corporación Humanas, 2009, p. 7).

Las mujeres que brindaron sus testimonios para esta investigación fueron agredidas sexualmente mediante coacción. Además, es preciso aclarar que dos de ellas sufrieron abuso sexual recurrente, tres fueron violentadas en un único hecho y dos tuvieron más de un agresor.

Después de un evento traumático como la VS, surge una transformación de significado del cuerpo en la mujer sobre todo en los aspectos cognitivo- afectivos, ya que estos van ligados a lo emocional, es decir, cómo se siente la mujer 
con su cuerpo. Para profundizar en los efectos que al nivel emocional generó la VS sobre las mujeres entrevistadas, se requiere examinar el significado que desde la psicología se ha otorgado a los términos emoción y sentimiento, puesto que se busca analizar cómo se presentan en el cuerpo de las mujeres a partir de los eventos traumáticos que sufrieron. La emoción es definida por Natalia Consuegra como:

Estado afectivo; reacción subjetiva al ambiente, acompañada de cambios orgánicos (fisiológicos y endocrinos) de origen innato, influida por la experiencia y que tiene la función adaptativa. Se refiere a estados internos como el deseo o la necesidad que dirige al organismo. La palabra emoción significa literalmente estado de excitación o estremecimiento. Suelen distinguirse tres componentes en cada emoción: un sentimiento característico o experiencia subjetiva, un esquema de estimulación fisiológica y un esquema de expresión abierta. El componente subjetivo es comunicado en las etiquetas que se le pone a las emociones, como temor, ira, gozo o tristeza. Las emociones cumplen un papel central en la gestión de procesos de autoprotección y autorregulación del organismo frente a situaciones extremas (2010, p. 92).

Sumado a lo anterior, la autora distingue dos tipos de emociones, a saber, las emociones primarias o automáticas que son la ira, el miedo, la alegría, el amor, la sorpresa, el disgusto, el interés y la tristeza; mientras que las emociones secundarias hacen referencia a las recién mencionadas cuando no son procesadas correctamente ante un estímulo determinado, lo cual hace que se produzcan otras derivadas de las primarias; dichas conversiones pueden ser: ira-rencor, miedo-ansiedad, tristeza-depresión, etc.

Como puede observarse en la afirmación "Me sentí traicionada y con mucha ira con mi novio, por eso me vengué y le pegué unas puñaladas, pero a pesar de eso mi ira no disminuyó, antes creció más" (Rosario, junio 21 de 2016), la mayoría de las mujeres entrevistadas, expresan en su mayoría emociones primarias, ira en una primera etapa, luego, a esta emoción la acompañan la tristeza y el miedo.

El sentimiento se refiere a un "sistema organizado de disposiciones emocionales que se centran alrededor de la idea de algún objeto. Según Jung, es una de las funciones o maneras de lidiar con el mundo. Es el acto de sentir, como el de pensar. Es una cuestión de evaluación de la información" (Consuegra, 2010, p. 242). Según lo anterior, los términos emoción y sentimiento son determinantes para el análisis del impacto que tuvo la vivencia de abuso sobre el cuerpo de las mujeres que hacen parte de esta investigación.

Las mujeres entrevistadas refirieron experimentar un sentimiento de venganza que se desprende de la ira producto de la VS. Este deseo de devolver al agresor el daño recibido se convierte en una manera de sobrellevar los efectos de la vivencia. Para Susana Velásquez (2003), las ideas de venganza provienen del odio generado por el sufrimiento que ha ocasionado el hecho violento; en 
este caso, el pensamiento o conducta vengativa genera "[...] un nuevo circuito de violencia (más de lo mismo) que realimentará la situación traumática para una víctima" (Velásquez, 2003, p. 103). Según lo anterior, el deseo de venganza, más que ayudar en la elaboración de lo ocurrido, mantiene a la mujer violentada atada a quien abusó de ella y al recuerdo del suceso traumático, es decir, la suspende en el tiempo como una víctima.

Al indagar por el estado de salud posterior al abuso sexual, las cinco mujeres entrevistadas indicaron que hubo un impacto sobre este; una de ellas expresó: "me sentía con una herida que me atravesaba durante los primeros seis meses" (Laura, junio 22 de 2016); dicha afirmación denota no solo las heridas y el daño en los genitales producto de la agresión, sino también el profundo daño emocional que experimentó en los meses posteriores al ataque sufrido.

El bienestar físico, mental y emocional de este grupo de mujeres se vio afectado significativamente: lesiones en los órganos sexuales y extremidades, dolor en los senos y los ovarios, daños en la matriz, caída del cabello, cefalea, opresión en el pecho, hipertensión, insomnio, pérdida del deseo sexual, ideación suicida, intentos de suicidio, depresión, trastornos de ansiedad, estrés postraumático, paranoia, trastorno afectivo bipolar, ciclos menstruales irregulares, alcoholismo y drogadicción fueron las consecuencias que mencionaron las mujeres que participaron de esta investigación sobre este tema en particular.

David Le Breton (1999) afirma que

El dolor quiebra la unidad vital... que tan evidente resulta cuando goza de buena salud, y confiando en sus fuerzas, olvida las raíces físicas de su existencia, cuando ningún obstáculo se interpone entre sus proyectos y el mundo. En efecto, en la vida cotidiana el cuerpo se vuelve invisible, dócil; su densidad se difumina en la ritualidad social y en la incansable repetición de situaciones cercanas unas de otras. El hecho de que el cuerpo escape a la atención del individuo condujo a René Leriche a definir la salud como "la vida en el silencio de los órganos". Georges Canguilhem añade que es un estado de "inconsciencia en que el sujeto pertenece a su cuerpo" (pp. 23-24).

Lo anterior permite hacer una lectura del sufrimiento experimentado por las mujeres entrevistadas durante y después del ataque, ya que con el hecho violento se desdibujaron los límites de su corporalidad, se rompió lo inscrito sobre su cuerpo durante su trayectoria vital y la naturalidad con la que se asumía la cotidianidad. El dolor surge cuando "la conciencia se descubre encerrada en las fronteras de un cuerpo en cuyo reconocimiento fracasa, pero que le impone su presencia" (Le Breton, 1999, p. 25), e implica un cambio en las relaciones sociales ya que se sobrelleva de dos maneras: por una parte, se hace a un lado el control que regularmente media la interacción con los otros, por lo tanto, la persona se permite expresar la afección que siente mediante el llanto, quejas, etc.; de otro lado, se encuentra el encierro en sí misma por la sospecha de no 
ser comprendida o para evitar un contacto con el que se pueda ver debilitada la relación con los allegados.

Es pertinente entonces, retomar los aportes de Juan Ramírez (2003), quien sostiene que las enfermedades tienen un componente biopsicosocial, según el cual, el dolor sería indicio tanto de causas orgánicas, como de las historias personales y las relaciones que se establecen en un contexto determinado.

[...] el cuerpo al sufrir dolor, al enfermar, comunica que en el entorno suceden otros dolores, éstos sociales, que por su parte afectan la salud del individuo, de donde la enfermedad es el mensaje que señala dicha dolencia colectiva. El cuerpo no solo es tejido que obedece a reglas biológicas, el cuerpo percibe, significa y comunica las contradictorias experiencias sociales humanas; en este contexto, el dolor, entendido desde los senderos nerviosos y los reflejos corporales no cuentan el relato completo sobre él mismo. De aquí que la enfermedad, bajo el lenguaje del dolor y el sufrimiento, elabora un texto donde el ser humano escribe un mensaje que da cuenta tanto de la bio-logía como de la bio-grafía de ese ser enfermo que sufre en sociedad (Ramírez, 2003, p. 214).

También en las entrevistas, al preguntar cómo son las relaciones con las demás personas que las rodean, se notó que la gran mayoría de las mujeres tomaron distancia de las relaciones interpersonales; la soledad, ahora, es una de las constantes para ellas: "yo ahora no tengo amigos ni nada solo voy de la casa al trabajo y mis hijos, esta es mi vida" (Julia, 21 de junio). Esto nos hacer evocar a Ramírez (2003) cuando hace referencia:

[...] El dolor somático va de la mano del dolor por las ausencias, el aislamiento, el distanciamiento de sus contactos humanos, de sus relaciones sociales con sus intercambios materiales y afectivos (Ramírez, 2003, p. 220).

Sobre la enfermedad mental se encontró que dos de las entrevistadas fueron tildadas de enfermas mentales, una por una funcionaria pública y otra por un familiar, como modo de restar importancia al acto criminal del que habían sido víctimas y de menoscabar sus requerimientos de protección y justicia. Cuando se preguntó sobre los cambios que se presentaron en su apariencia corporal después de sufrir el episodio de VS, tres mujeres manifestaron haber modificado su cabello, María lo cortó debido a la asociación que hacía de este con el hecho traumático, ya que fue por donde el agresor la sujetó para posteriormente violentarla, por lo que al tomar esa determinación, intentó eliminar dicho recuerdo y un factor que la hacía vulnerable; Liliana indicó que teñía su cabello "tapando esa mugre con la que se queda" (Liliana, junio 27 de 2016). Por último, Rosario mencionó que antes del suceso llevaba el cabello corto, pero luego de este, lo dejó crecer pues quería llamar la atención de los hombres debido a que comenzó a ejercer la prostitución. 
María, Rosario y Laura indicaron haber adelgazado después de haber sido abusadas, lo cual asociaron a su estado ansioso-depresivo y a una inherente pérdida del apetito. Por su parte, Julia y Liliana experimentaron un aumento de peso relacionado con el estrés y la ansiedad. Los cambios generados en su apariencia por la pérdida y el aumento de peso tuvieron un impacto negativo en la apreciación que tenían estas mujeres de su imagen corporal, tal y como lo demuestra la siguiente afirmación: "Perdí peso, no comía bien. Me sentía mal al mirarme al espejo y ver lo que había quedado de mí" (Laura, junio 22 de 2016).

\section{Reivindicación de las mujeres sobrevivientes}

Para Deysy Zuluaga y Alfonso Insuasty (2011), la generación de conocimiento enmarcado en el modelo occidental parte de una visión homogénea, centralista, universal y excluyente, lo cual se ha fundamentado en enfoques metódicos, demostrables, comprobables, objetivos y racionales, cuyos resultados adquieren las cualidades de verdadero y científico. Según esta lógica, los demás saberes carecen de validez; tal es el caso del conocimiento de las mujeres, puesto que obedece al campo del "sentir" que no pasa por el filtro de la razón.

Lo anterior permite introducir la crítica que la epistemología feminista ha hecho al método científico por excluir la subjetividad de la producción de conocimiento; de manera opuesta, la investigación feminista permite incorporar las experiencias de las mujeres, incluir explicaciones de los fenómenos que afectan sus vidas y situar a las investigadoras en el mismo lugar que al objeto de estudio. Las anteriores son características del punto de vista feminista, metodología que según Sandra Harding (1987),

[...] contrasta con la forma usual que da origen a los proyectos de investigación en las ciencias sociales o naturales, con los problemas que plantean las disciplinas, las corporaciones, los gobiernos, las agencias de ayuda internacional y otras instituciones de cuyos diseños las mujeres han sido, en su mayoría, excluidas. Esos nuevos "métodos" feministas han generado preguntas sobre, por ejemplo, la doble jornada de trabajo de las mujeres, la contribución del trabajo doméstico a la economía, la violencia sexual o las formas de organización política que prefieren las mujeres. Las respuestas a estos interrogantes usualmente no pueden ser encontradas al inspeccionar la vida de las mujeres, ya que la vida de estas se organiza lejos de las formas en que las disciplinas recogen y organizan la información, y de las políticas gubernamentales, de las corporaciones o de otras instituciones (p. 11).

Esta metodología empleada permitió analizar el fenómeno de la VS desde la perspectiva de las experiencias de estas cinco mujeres, teniendo en consideración las diferencias existentes entre las personas entrevistadas, es decir, sus edades, condición socioeconómica, nivel de estudios, etc. Y, a su vez, brindó a las investigadoras la oportunidad de aportar a la reflexión desde su comprensión 
al nivel tanto personal como académico, ya que la generación de conocimiento desde el punto de vista feminista no puede desligarse del contexto en el que se inscribe quien realiza la investigación.

En los relatos de las entrevistadas se aprecia que sus cuerpos no son únicamente el territorio sobre el cual se perpetró la acción violenta y en el que se manifestaron las secuelas de la misma, sino que, además, constituye un lugar de resistencia. Las mujeres que han sufrido VS exhiben una lucha donde puede apreciarse la fuerza y capacidad de sus cuerpos para hacer frente a la experiencia dolorosa y activar mecanismos que permitan transformarla en nuevos sentidos para su existencia. Por lo anterior, es pertinente reflexionar sobre la diferencia existente entre víctima y sobreviviente que enuncia Susana Velásquez (2003), ya que el término víctima puede asociarse a una condición de pasividad, mientras que la expresión sobreviviente remite al conjunto de acciones que permiten a la persona violentada transformar su realidad mediante la concienciación de lo ocurrido, asumir una postura de resistencia y emprender el camino a su recuperación.

Para Velásquez (2003), existen dos posibles consecuencias del acto violento sobre la identidad de la mujer atacada, ya que esta puede tomar lo sucedido como un acontecimiento que hace parte de su historia personal, o puede asumir su identidad en función del suceso; esta última tiene la implicación de terminar convirtiéndola en una víctima para siempre, es decir, cuando la mujer que ha sido agredida se asume como una sobreviviente encuentra allí un mecanismo para trascender el hecho, comprendiendo que el acto violento cometido sobre su integridad no constituye su existencia, y así, reconstruir su vida.

Por su parte, Patsilí Toledo (2012) prefiere hablar de mujeres que han vivido violencia que de víctimas, ya que la victimización obstaculiza su capacidad de agencia frente al suceso, atribuyéndole una actitud pasiva con respecto al delito y al proceso penal. Adicionalmente, aunque reconoce que es una expresión afirmativa, problematiza el concepto de sobreviviente, porque puede instaurarse una dicotomía entre víctima y sobreviviente o considerar que existen fases que debe atravesar quien vivió el hecho violento o conducir a que disminuya el presupuesto dispuesto para las mujeres que han sufrido estos actos, si se concibe una excesiva responsabilidad y empoderamiento de su parte. Para la autora, el discurso de la violencia contra las mujeres está institucionalizado a través del derecho, el cual ha reducido este fenómeno a un conflicto interpersonal, dejando de lado la responsabilidad del Estado y la sociedad en la materia. Asimismo,

El vocabulario terapéutico, junto con la comprensión popular de la violencia, hace que predomine una visión individualista de las mujeres agredidas, que las construye a ellas mismas como víctimas "individuales" y, a la vez, como "agentes de su propia 
liberación", es decir, quienes deben hacer el tránsito de víctimas a sobrevivientes. De alguna manera, las actitudes que muestran los agresores hacia las mujeres -al responsabilizarlas por la violencia y hacer que sean ellas quienes ajusten su comportamiento para evitarla-también se presentan en organizaciones y personas que actúan "a favor" de las mujeres: se espera que "ellas ajusten su comportamiento" para evitar la violencia, ingresando a refugios, asistiendo a terapia, perseverando en el proceso judicial, etc. A menudo, las mujeres que buscan ayuda profesional entran en un nuevo espacio de relaciones de poder, en donde pueden ser patologizadas como mentalmente inestables o incapaces de valorar su propia situación (Toledo, 2012, p. 58).

En el relato de Liliana, se aprecia esta problemática revictimizante donde se cruzan ambos discursos: "para la comisaria esto se volvió algo personal, me ha tildado de loca, tuve que ir donde un médico que certificara que yo no tengo una enfermedad mental" (junio 27 de 2016). Lo anterior muestra que los imaginarios que normalizan la violencia también se encuentran presentes en la institucionalidad, son causantes de que se reste importancia a las experiencias de estas mujeres, e incluso generan la justificación o la absolución de sus agresores.

Velásquez (2003) llama la atención sobre la respuesta que se genera en las comunidades donde se presentan casos de VS, enuncia el olvido como un mecanismo mediante el cual se lidia con los hechos violentos que causan displacer, lo cual propicia la repetición de los acontecimientos. El olvido que caracteriza a una población donde se aíslan este tipo de sucesos constituye un indicador de la desigualdad de género en nuestro contexto, ya que esta respuesta invisibiliza y naturaliza la violencia que se ejerce sobre las mujeres.

Hilderman Cardona (2016) afirma que la sociedad requiere una respuesta ética ante fenómenos como el de la violencia, ya que es una situación que implica un encuentro con quien es mi semejante, cuyo cuerpo fue destrozado, humillado, abusado, etc. Lo anterior revela el valor que se otorga a la dignidad de la vida humana en un determinado grupo social; por ende, que se precisen explicaciones sociales, ya que la VS tiene su origen en unas relaciones de poder que se reflejan en una forma de dominación que se ha naturalizado en la sociedad.

La VS es un tema tabú puesto que la sexualidad de las mujeres se encuentra enmarcada en unos roles culturalmente establecidos, motivo que conduce a su señalamiento y estigmatización, haciendo que se sientan culpables y avergonzadas, en vez de apoyadas y protegidas. En el caso del abuso sexual infantil, también existe un tabú puesto que alude a un tipo de violencia cometida sobre una persona que por su edad precisa de una política y estética del cuidado; además, en aquellos casos donde los agresores son familiares, como en los de esta investigación, involucra la transgresión de la prohibición del incesto². 2 De acuerdo con Claude Lévi-Strauss (1969), la prohibición del incesto es la única regla social que posee un
carácter universal, surgió con la finalidad de conformar alianzas entre grupos mediante el intercambio de 
Durante esta investigación, se contó con el testimonio de dos mujeres que vivieron VS siendo menores de edad, y de otra, cuya hija de tres años fue abusada por su esposo y, a la vez, padre de la menor. María expresó en su relato: “Una compañera del colegio supo y le dijo a un docente, hicieron un consejo directivo y nadie me creyó. Entonces todo se supo y empecé a vivir algo terrible porque hasta los vecinos se burlaban de mí" (julio 7 de 2016).

Ámbitos escolares, universitarios, familiares, vecinales y jurídicos fueron mencionados por las entrevistadas como espacios donde se presentaron algunas de las siguientes situaciones: omisión de medidas para enfrentar el hecho violento, desestimación de la experiencia y requerimientos de quien vivió el acontecimiento, así como señalamiento y maltrato de la mujer abusada. Estos lugares, que pudieron convertirse en entornos protectores, se transformaron en ámbitos donde predominaron el silencio y / o la impunidad, desaprovechando la oportunidad de realizar una reflexión sobre lo ocurrido.

Expresiones como: "eso le pasó por estar vestida así”, "una mujer precavida no hubiera estado sola por ahí", "es que estaba borracha", "¿qué hombre puede resistirse ante una mujer así?", "no hay que meterse en asuntos ajenos" y "¿qué tiene de malo si es el novio / marido?". Esta serie de juicios persisten en las comunidades donde acontecen casos de VS, justifican e invisibilizan la problemática tratada y constituyen la reafirmación de las relaciones de poder presentes en la sociedad.

Nuestra actualidad exige asumir frente al mundo una condición guerrera, experimental. La guerra es crear nuevos valores que afirmen la vida. Estamos obligados a experimentar con situaciones inéditas. Por esto, la crueldad como problema deriva en actos creadores. Tenemos que inventarnos las reglas del juego, proponer y crear nuevas realidades. Por esto, la vida tiende a parecerse a una práctica artística generalizada: el guerrero que ha pasado por la crueldad, es un creador de valores, un artista que produce nuevas realidades y al hacerlo, produce vida. La guerra cruel consiste en crear estrategias para afirmar la vida en un momento en el que existen las condiciones para perecer a cada instante (Pabón, 2002, p. 63).

Sobre el cuerpo humano recaen poderes que lo limitan, censuran, maltratan y oprimen, pero también es un lugar desde el cual es posible la transmutación y la generación de nuevos valores culturales, por lo que el despliegue de la maquinaria guerrera de los cuerpos que menciona Consuelo Pabón (2002), es su capacidad de resistir. "El intento será no dejarnos fulminar en esa condición de caos, sino conocer desde ahí lo que puede nuestro cuerpo, cómo se resiste, cómo

mujeres y constituye el paso decisorio de la naturaleza a la cultura. Para mayor información, consultar los textos Las estructuras elementales del parentesco y El tráfico de mujeres: Notas sobre la "economía política" del sexo. 
es capaz de realizar hazañas inimaginables para sobrepasar lo insoportable" (p. 64).

Cuando Liliana dice: "Yo no vivo más de tres meses en la misma parte porque siento mucho temor" (junio 27 de 2016), está haciendo referencia a una vida que transcurre al límite, bajo el acecho de quien la violentó tanto a ella como a su hija; por lo tanto, implicó la búsqueda de mecanismos que aseguren la integridad de ambas, ya que en instancias institucionales no ha conseguido la protección necesaria. Lo anterior-es evidencia de la existencia en pie de guerra que debe asumir esta mujer en su día a día. Pabón (2002) sostiene al respecto:

A nivel del espacio, lo que se vive es una desterritorialización, un abandono del territorio que daba seguridad con sus límites. Los cuerpos se ven obligados a abandonar su condición sedentaria y nomadizarse; por fuera de la voluntad de un sujeto, el cuerpo tiene que romper rutinas y costumbres y adaptarse a las circunstancias (p. 66).

Otro espacio desde el cual se observa la lucha de estas mujeres a partir de su corporeidad se aprecia en el testimonio de María, la cual se refiere a esta como una vía de resistencia y transmutación de la violencia a la que fue sometida en repetidas ocasiones, manifestando en sus palabras un trabajo de historización del propio cuerpo, comprensión del papel que tiene la educación en la prevención de la VS, incorporación de la sensibilidad de género y reconocimiento de la corporalidad como espacio de trabajo terapéutico.

Desde que empecé a venir a CERFAMI, he mejorado mucho, yo hice un trabajo para verme al espejo y descubrir mis cicatrices. Las terapias de CERFAMI me han ayudado mucho, incluso estoy haciendo un programa para trabajar con jóvenes sobre el tema de respetarse [...]. Hay que reeducar a los jóvenes, enseñarles sobre el respeto por el cuerpo, enseñar que la mujer no es un objeto, prevenir el embarazo en adolescentes. Yo tengo muchas precauciones al tocar los genitales de mis hijos. Es importante hacer un ejercicio donde se observe el propio cuerpo, emplear cartografía humana, reconocer en el cuerpo la propia historia de vida (julio 7 de 2016).

María mencionó en su relato diferentes estrategias para otorgar un nuevo sentido a sus experiencias; afirmó que encontró en la escritura un medio para desahogarse, mientras que el trabajo que ha realizado para reconocer la historia de su propio cuerpo le ha permitido comprender los diferentes efectos de la VS sobre el mismo; por ejemplo, manifestó haber desarrollado una limitación en su movimiento y expresión corporal a raíz de los abusos vividos. Esta consecuencia llama la atención, porque el movimiento se relaciona con la acción y la relación que se establece con el entorno donde se desenvuelve la existencia.

Espacios como CERFAMI donde se realiza un trabajo con el cuerpo permiten a quienes han vivido VS reconocer y transformar sus heridas mediante 
acciones individuales y grupales; dentro de estas acciones se encuentran: el ámbito terapéutico, la escritura, la cartografía humana, los círculos de mujeres y las expresiones artísticas. Los anteriores son medios que permiten recorrer nuevos caminos ante la experiencia vivida, brindan la oportunidad de manifestar el dolor, resistir ante la violencia, establecer nuevos vínculos, buscar la reapropiación del cuerpo, expresar la creatividad, crear memoria y, como en el caso de María, transformarse en una agente activa que genere conciencia sobre la materia.

En el contexto colombiano la violencia contra las mujeres se manifiesta tanto en la esfera privada como en la pública; en esta última, sobresale el conflicto armado, en el cual, a la vez que se reconoce un aspecto de dominio de territorios al nivel nacional, se suma el acceso no solo topográfico, sino del cuerpo de la población en situación de vulnerabilidad. La VS, según Sonia Fiscó (2005), ha sido una táctica de guerra que se ha empleado con la intención de calmar o premiar a las tropas, humillar al enemigo y tomar a las mujeres como botín de guerra. Para la autora, aunque esta ha sido una historia invisibilizada en los documentos oficiales, no se puede negar que "las mujeres, las jóvenes y las niñas sufren y han sufrido los efectos directos e indirectos de la confrontación en las distintas etapas de nuestra historia de violencia sociopolítica" (p. 126).

De manera que el conflicto armado constituye otro ámbito en el que debe resaltarse la resistencia y reconocer las experiencias de las mujeres que han vivido VS. Como afirma Kevin García (2016),

Existen seres extraordinarios en la cotidianidad de nuestras regiones. Ciudadanos que lograron superar el asesinato de seres queridos, el maltrato físico y psicológico, el abandono estatal, el encierro y encadenamiento. Colombianos que como los guaduales lograron soportar las tempestades del conflicto sin romperse. Resistieron y luego reinventaron sus vidas en medio de las ruinas, forjaron proyectos y asumieron causas de reparación del tejido social. Relatos de sobrevivencia, construcción de sentido de vida, formas de poetización de la existencia, de construcción de razones para seguir viviendo (p. 142).

Según este autor, la importancia de la construcción de memoria histórica, verdad y reparación radica en que posibilita la disminución de la pulsión por la violencia, situar el tema en la agenda pública, dar visibilidad a los hechos y presionar a los entes judiciales para la entrega de resultados en los procesos que adelantan.

\section{Conclusiones}

Esta investigación permitió reconocer en las historias de quienes han vivido VS un llamado a la dignidad humana, una exhibición de resistencia y lucha, así 
como la demostración de la capacidad que tienen las personas para sobreponerse ante la adversidad. De igual manera, se destaca el potencial que tiene el trabajo desde el propio cuerpo para su rehabilitación, generando la posibilidad de retomar un lugar en el mundo a partir del fortalecimiento que surge del trabajo de elaboración del suceso, para finalmente inscribirlo en un contexto más amplio de la propia vida.

El conjunto de mujeres que participaron en esta investigación conforma una serie de experiencias encarnadas de la violencia; es decir, en su corporalidad se evidencia la experiencia del dolor surgida a partir del hecho violento, a la par que ven modificado su habitus de género cuando se transforma su corporalidad como consecuencia del acto abusivo, ya sea en su comportamiento o en su imagen corporal. Las construcciones cargadas de sentido hasta dicho momento se modifican debido a que surgen una serie de cambios que no concuerdan con su rol en la sociedad ni con los ideales de belleza establecidos en su contexto cultural, llevándolas a sentir intranquilidad, inseguridad y, en ocasiones, desprecio por su cuerpo.

Los principales cambios que tuvieron lugar en sus cuerpos a partir de la experiencia fueron: aumento o pérdida de peso, desarrollo de trastornos psicosomáticos, variaciones en la expresión corporal, desidia con relación al arreglo personal y ejecución de las actividades acostumbradas, aislamiento, sensación de encontrarse sucia y sentimiento de miedo, angustia y vulnerabilidad.

En torno a la VS se encontraron concepciones ideológicas que, como en el caso de las creencias religiosas de Julia, influyeron sobre la decisión de continuar un embarazo resultado del hecho violento. La expresión: "a un loco no hay que provocarlo", proferida por la misma mujer, constituye una justificación de este fenómeno; además, tanto Laura como Julia refirieron extrañeza en el hecho de haber sido agredidas sexualmente a su edad, denotando la idea de que solo sobre las jóvenes pueden cometerse estos delitos.

La mayoría de mujeres que participaron en esta investigación se hallan o han estado en algún momento de su vida en un ciclo de la violencia donde repetidamente se afecta su autoestima y capacidad para dar solución a una situación conflictiva. Debe resaltarse que esta violencia ha sido en ocasiones ejercida por las parejas que conocieron después de haber vivido los hechos violentos, y quienes las han ayudado en la superación de los mismos; en estos casos, daño psicológico y físico fueron los mencionados al momento de la entrevista. Ante dicha situación surge la pregunta: ¿impide el rol de género aprendido establecer relaciones entre hombres y mujeres donde no tenga lugar la violencia? 


\section{Referencias bibliográficas}

Bordieu, Pierre (1993) [1980]. El sentido práctico. Madrid: Taurus.

Bordieu, Pierre (2000) [1998]. La dominación masculina. Barcelona: Anagrama.

Cardona, Hilderman (2017). Testimonios visuales de la violencia: el cuerpo deshecho. En Narrativas corporales de la violencia y estéticas del dolor. Medellín-México: Universidad de Medellín y La Cifra Editores.

Cardona, Hilderman, Sánchez, Natalia (2012). Cuerpos degenerados y conductas anormales: la transgresión a las sexualidades legitimadas en Medellín entre 1957 y 1966. Ciencias Sociales y Educación, $1,37-60$.

CERFAMI (2014). Documento interno de la Corporación sobre los 25 años de su fundación.

Consuegra, Natalia (2010). Diccionario de psicología. Bogotá: Ecoe.

Corporación Humanas Colombia (2009). Situación en Colombia de la violencia sexual contra las mujeres. Recuperado de http://www.humanas.org.co/archivos/Situacion_en_Colombia_de_la_violencia_sexual_contra_las_mujeres.pdf

Duch, Lluís, Mèlich, Joan (2005). Escenarios de la corporeidad: antropología de la vida cotidiana. Madrid: Trotta.

Fiscó, Sonia (2005). Atroces realidades: la violencia sexual contra la mujer en el conflicto armado colombiano. Papel Político, 17, 119-159.

García, Kevin (2016). Cubrimientos periodísticos en reconstrucción de memoria. En Pistas para narrar la memoria: periodismo que reconstruye las verdades (134-161). Bogotá: KAS.

Harding, Sandra (1998). ¿Existe un método feminista? Traducido por Gloria Elena Bernal. Recuperado de http://bidi.xoc.uam.mx/tabla_contenido_libro.php?id_libro=81

Le Breton, David (2002). La sociología del cuerpo. Buenos Aires: Nueva visión.

Le Breton, David (1999). Antropología del dolor. Barcelona: Seix Barral.

Lévi-Strauss, Claude (1969). Las estructuras elementales del parentesco. Barcelona: Paidós.

Pabón, Consuelo (2002). Construcciones de cuerpos. En Expresión y vida: prácticas en la diferencia (36-79). Bogotá: Escuela Superior de Administración Pública.

Pérez, Yovana (2010). La atención integral a mujeres víctimas de violencia sexual, un compromiso interinstitucional en la ciudad de Bogotá D. C. Tesis de Maestría en Política Social de la Pontificia Universidad Javeriana. Recuperado de http://repository.javeriana.edu.co/bitstream/10554/875/1/ pol155.pdf

Puleo, Alicia (2015). Ese oscuro objeto del deseo: cuerpo y violencia. Revista Investigaciones Feministas, $16,122-138$.

Ramírez, Juan (2003). Cuerpo y dolor: semiótica de la anatomía y la enfermedad en la experiencia humana. Toluca: Universidad Autónoma del Estado de México.

Seoane, Elisa (2015). Erotismo y pornografía en el cómic underground hecho por mujeres. Análisis crítico desde la perspectiva de género de la invisibilidad de la autoría femenina del comix erótico-pornográfico 
feminista. Tesis doctoral en Filosofía de la Universidad de Sevilla (España). Recuperado de https:// idus.us.es/xmlui/handle/11441/34223

Toledo, Patsilí (2012). ¿Vivir violencia y/o ser víctimas? Noviembre 21 de 2016, de Red Chilena contra la Violencia Doméstica y Sexual. Sitio web: http://www.nomasviolenciacontramujeres.cl/wpcontent/uploads/2015/11/mujeres_y_violencia_silencios_y_resistencia.compressed.pdf

Velásquez, Susana (2003). Violencias cotidianas, violencia de género: escuchar, comprender, ayudar. Buenos Aires: Paidós.

Zuluaga, Deysy, Insuasty, Alfonso (2011). Metodología feminista en la investigación: el reto epistemológico del cuerpo. Revista Venezolana de Estudios de la Mujer, 16, 43-54. 
